Regular Paper

\title{
Dynamic Analysis of Latch-in Relay Using 3-D Finite Element Method with Mesh Modification Method Employing Multi-Mesh and the Interpolation
}

\author{
T. YAMAGUCHI ${ }^{* 1}$, Y. KAWASE $^{* 1}$ and T. ASANO ${ }^{* 1}$
}

\begin{abstract}
This paper describes a new mesh modification method for the three-dimensional finite element method, which prevents the mesh being broken to achieve the movement of the armature with long stroke. The effectiveness of this method is clarified through the dynamic characteristics analysis of a latch-in relay.
\end{abstract}

Keywords: 3-D finite element method, latch-in relay, multi-mesh modification method, interpolation. (Received: 31 May 2012, Revised: 10 June 2013)

\section{Introduction}

In the industrial field, various kinds of dynamic characteristics analyses using the three-dimensional finite element method (3-D FEM) are achieved for the optimal design of electrical apparatus. In these analyses, various mesh modification methods have been reported to achieve the movement of the armature [1-3].

In Ref. 2, two meshes are prepared, and the movement of the armature is achieved by interpolating the coordinates of nodes from two meshes. In Ref. 3, the appropriate mesh is chosen from the meshes prepared in advance and the coordinates of nodes are modified by solving the Laplace equation. However, it is not easy to simulate the dynamic behavior of the armature with long stroke even if those methods are used.

In this paper, we combine those mesh modification methods to achieve the movement of armature with long stroke, which prevents the mesh being broken. Our new method is applied to a latch-in relay. The availability of this method is clarified through the dynamic characteristics analysis of a latch-in relay.

\section{Analysis Method}

\subsection{Magnetic Field Analysis}

The equations of the magnetic field and the electrical circuit are coupled with the use of the 3-D FEM, which are given by the magnetic vector potential $\boldsymbol{A}$ and the exciting current $I_{0}$ as follows:

$$
\begin{aligned}
& \operatorname{rot}(v \operatorname{rot} \boldsymbol{A})=\boldsymbol{J}_{0}+v_{0} \operatorname{rot} \boldsymbol{M} \\
& E=V_{0}-R I_{0}-\frac{d \psi}{d t}=0
\end{aligned}
$$

\footnotetext{
Correspondence: Y. KAWASE, Department of Information Science, Gifu University, 1-1, Yanagido, Gifu, 501-1193, Japan email: kawase@gifu-u.ac.jp

${ }^{* 1}$ Gifu University
}

$$
\boldsymbol{J}_{0}=\frac{n_{c}}{S_{c}} I_{0} \boldsymbol{n}_{S}
$$

where $v$ is the reluctivity, $\boldsymbol{J}_{0}$ is the exiting current density, $v_{0}$ is the reluctivity of vacuum, and $\boldsymbol{M}$ is the magnetization of the permanent magnet, $V_{0}$ is the applied voltage, $R$ is the effective resistance, $\psi$ is the interlinkage flux of the exiting coil, $n_{\mathrm{c}}$ is the number of coil turns, $S_{\mathrm{c}}$ is the cross-sectional area of the coil and $\boldsymbol{n}_{\mathrm{s}}$ is the unit vector along with the direction of exciting current.

The interlinkage flux $\psi$ is written using the magnetic vector potential $\boldsymbol{A}$ as follows:

$$
\psi=\frac{n_{c}}{S_{c}} \int A n_{s} d v
$$

Solving Eq. (4) with the step-by-step method, $d \psi / d t$ in Eq. (2) is written as follows:

$$
\frac{d \psi}{d t}=\frac{\psi^{i}-\psi^{i-1}}{\Delta t}
$$

where $i$ is step, and $\Delta t$ is the time interval.

$d \psi / d t$ in Eq. (5) can be calculated even if the mesh is changed between steps $i$ and $i-1$.

\subsection{Motion Equation}

The motion equation of the armature in the latch-in relay is described as follows:

$$
I \frac{d^{2} \theta}{d t^{2}}+k \theta+T_{s}=T_{\theta}
$$

where $I$ is the moment of inertia of the armature, $\theta$ is the rotation angle, $k$ is the spring constant, $T_{s}$ is the initial spring force and $T_{\theta}$ is the torque acting on the armature.

\subsection{Mesh Modification Method by the Interpolation}

In the method proposed in Ref. 2, two meshes are prepared as shown in Fig. 1. One is the initial mesh, which gives the initial shape and another is the final mesh, which gives the final shape. The numbers of elements and nodes in the initial mesh are equal to those 
in the final mesh, respectively. In addition, the nodes which constitute elements in the initial mesh are the same as those in the final mesh. Only the coordinates of the nodes are different. These meshes can be facilitated only by changing the position of the armature when the 3-D meshes are generated automatically.

The new coordinate $Q_{\mathrm{P}}$, values of each node after the movement of the armature are determined by interpolating between the initial coordinate $Q_{\mathrm{P}}$ and the final coordinate $Q_{\mathrm{P}}$, as follows:

$$
Q_{\mathrm{P}^{\prime \prime}}=R Q_{\mathrm{P}^{\prime}}+(1-R) Q_{\mathrm{P}}
$$

where $R$ is the movement distance ratio which is defined as follows:

$$
R=\frac{\Delta l}{l} \quad(0 \leqq R \leqq 1)
$$

where $\Delta l$ is the movement distance obtained by solving Eq. (6) and $l$ is the distance from point $\mathrm{P}$ to $\mathrm{P}$ ' in Fig. 1. The motion problems can be solved by applying Eq. (7) to all nodes in the analysis region.

However, in the case to apply this method to long stroke movement of the armature, the accuracy of the analysis becomes worse because of flat elements in meshes.

\subsection{Proposed Mesh Modification Method}

Ref. 3 proposes the multi-mesh modification method, in which the appropriate mesh is chosen from the meshes prepared in advance, and then the coordinates of nodes are modified by solving the Laplace equation for achieving the movement of armature. However, in some cases, the mesh is broken in the air region between the stator and the armature.

We propose a new mesh modification method, which is combined the mesh modification methods mentioned above in order to achieve the movement of armature with long stroke.

Advantage of our new mesh modification method is twofold: Eq. (1) flat elements are not almost generated, and Eq. (2) no mesh is broken.

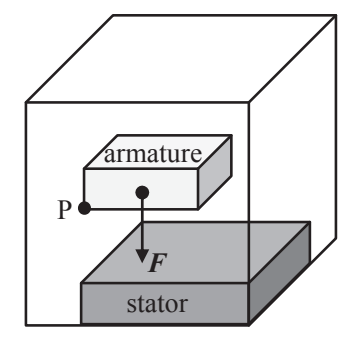

(a) initial shape

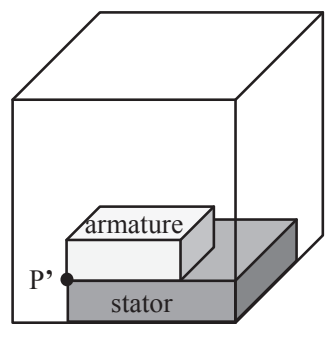

(b) final shape
Fig. 1. Interpolation.
Fig. 2 shows the flowchart of the dynamic analysis of a latch-in relay with our new mesh modification method. In this method, the range of the movement of the armature is divided into several zones, and two meshes of the initial mesh and the final mesh are prepared in each zone. The appropriate zone is chosen from these zones according to the rotation angle of the armature. The mesh for calculation is modified by interpolating the coordinates of nodes between the initial mesh and the final mesh in chosen zone. Therefore, it is possible to simulate the dynamic characteristics of movement of the armature with long stroke using this method. The procedure of this method is executed as follows:

(a) The 3-D magnetic field analysis is executed. The torque acting on the armature is calculated using the nodal force method [4].

(b) The rotation angle of the armature is calculated by solving Eq. (6).

(c) The appropriate zone is chosen from several zones according to the rotation angle of the armature.

(d) The mesh modification for the next time step is executed by interpolating the coordinates of nodes from the initial and the final meshes in the chosen zone.

\section{Analyzed Model and Conditions}

The proposed method is applied to the rotational movement of the armature of a latch-in relay.

Fig. 3 shows the analyzed model of a latch-in relay. This relay consists of an armature, a permanent magnet, a stator and a coil. The rotation angle is given by $\theta$ as shown in Fig. 3 (b). The analysis region is $1 / 1$ of the

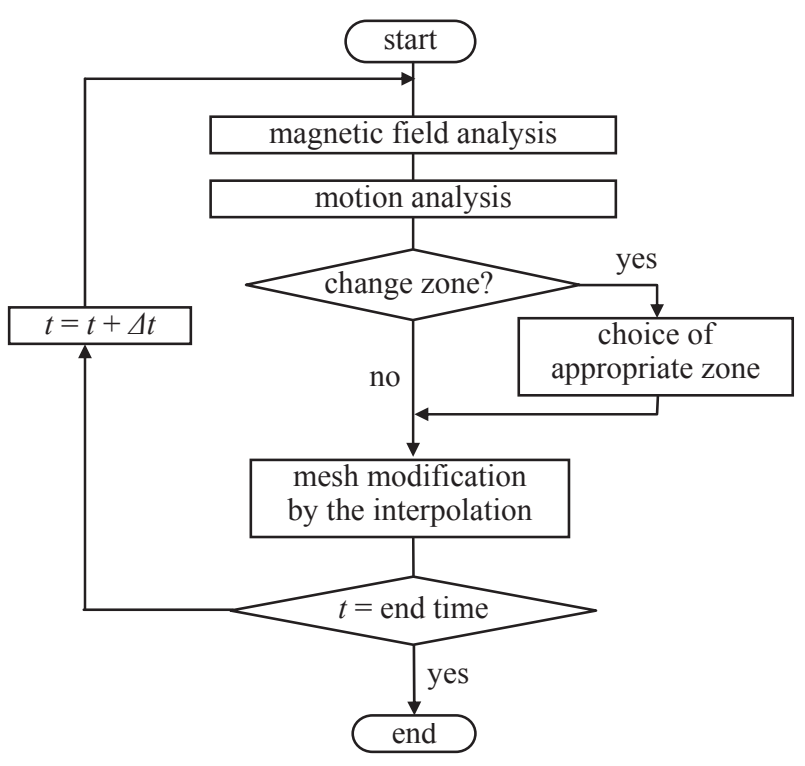

Fig. 2. Flowchart of dynamic analysis. 
whole region, because of the non-symmetrical shape of the model.

Fig. 4 shows finite element meshes when the rotation angle $\theta$ is in zone II as shown in Fig. 7. When the rotation angle $\theta$ is in zone II, the mesh for calculation is generated by interpolation from the two meshes.

The magnetic nonlinearity of the stator and the armature is taken into account. Fig. 5 shows the B-H curve for the stator and the armature.
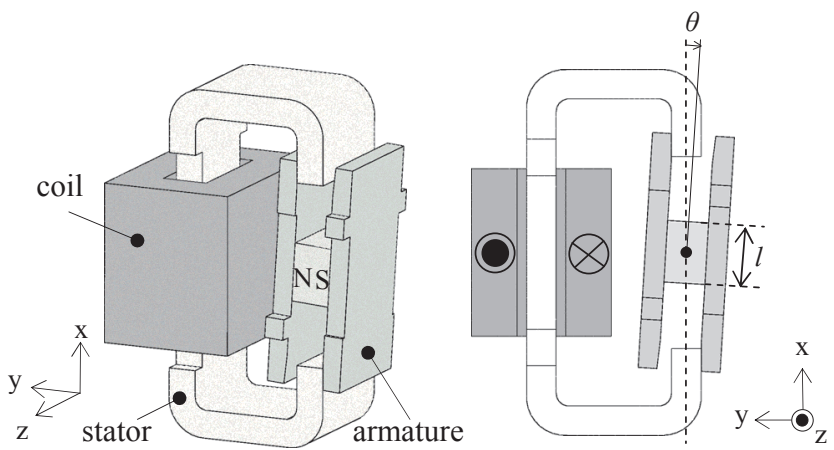

(a) overall view

(b) rotation angle $\left(\theta=-3.5^{\circ}\right)$

Fig. 3. Analyzed model.

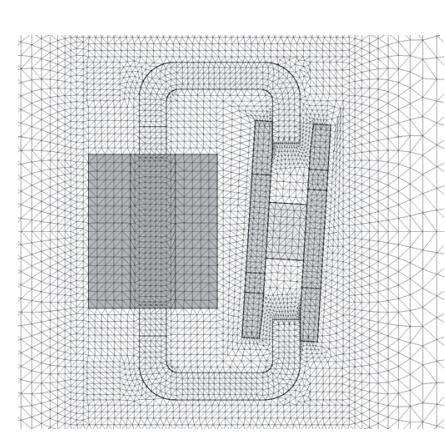

(a) initial mesh $\left(\theta=-3.0^{\circ}\right)$
Fig. 6 shows the characteristics of the spring load connected to the armature.

The range of the rotation angle of the armature is divided into three zones as shown in Table 1 and Fig. 7.

Table 2 shows the analysis conditions in dynamic analysis.

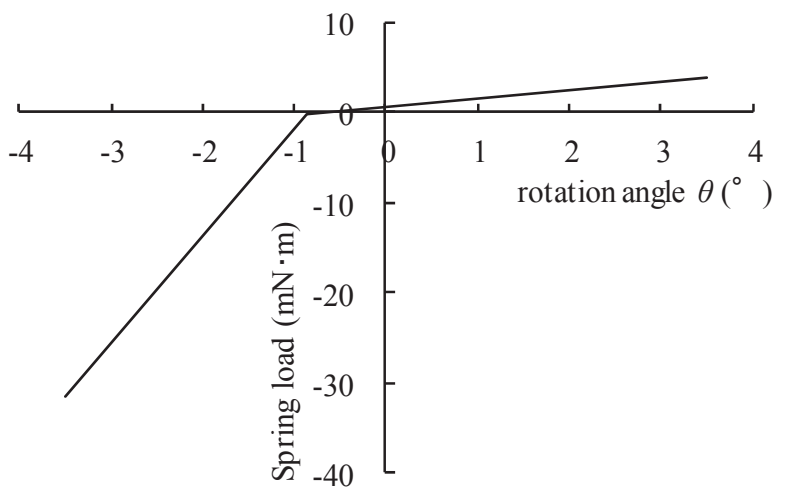

Fig. 6. Characteristics of spring load.

Table 1 Range of rotation of armature

\begin{tabular}{l|c}
\hline zone I & $-3.5^{\circ} \leq \theta<-3.0^{\circ}$ \\
\hline zone II & $-3.0^{\circ} \leq \theta<3.0^{\circ}$ \\
\hline zone III & $3.0^{\circ} \leq \theta \leq 3.5^{\circ}$ \\
\hline
\end{tabular}

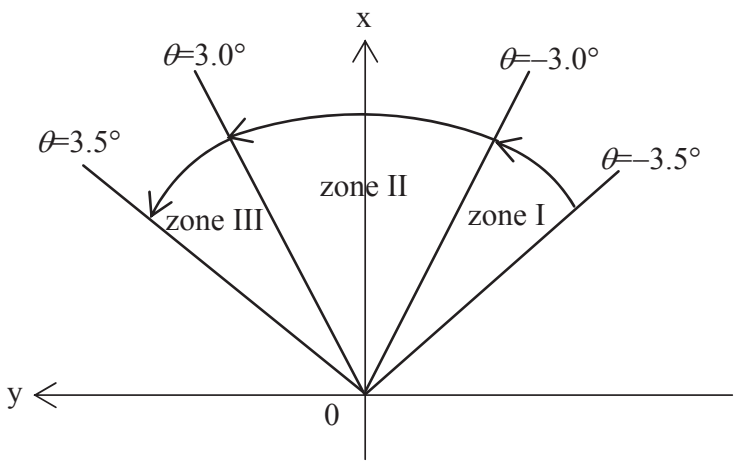

Fig. 7. Range of rotation of armature.

Table 2 Analysis conditions

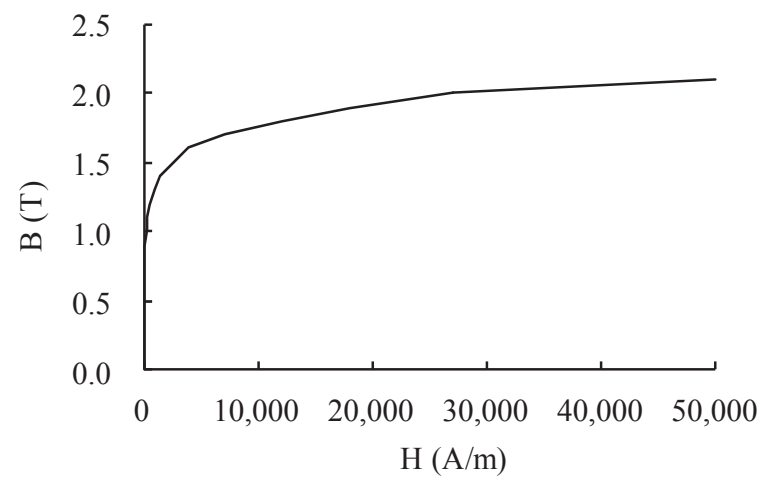

Fig. 5. B-H curve (SPCC).

\begin{tabular}{|c|c|c|c|c|}
\hline \multicolumn{2}{|c|}{ Time interval (ms) } & \multicolumn{3}{|c|}{0.1} \\
\hline \multirow[t]{2}{*}{ coil } & $\begin{array}{c}\text { Number of turns } \\
\text { (turn) }\end{array}$ & \multicolumn{3}{|c|}{676} \\
\hline & Resistance $(\Omega)$ & \multicolumn{3}{|c|}{10} \\
\hline \multicolumn{2}{|r|}{ Voltage (V) } & \multicolumn{3}{|c|}{4.5} \\
\hline \multicolumn{2}{|c|}{$\begin{array}{c}\text { Magnetization of } \\
\text { permanent magnet }(\mathrm{T})\end{array}$} & 0.430 & 0.645 & 0.860 \\
\hline \multicolumn{2}{|c|}{ Range of rotation $\left(^{\circ}\right)$} & \multicolumn{3}{|c|}{$-3.5 \leq \theta \leq 3.5$} \\
\hline \multicolumn{2}{|c|}{$\begin{array}{c}\text { Inertia of armature } \\
\left(\mathrm{N} \cdot \mathrm{m} \cdot \mathrm{s}^{2} / \mathrm{rad}\right)\end{array}$} & \multicolumn{3}{|c|}{$1.51620 \times 10^{-7}$} \\
\hline
\end{tabular}




\section{Comparison between Proposed Method and Conventional Interpolation Method}

In order to clarify the effectiveness of the proposed method, the static torque characteristics of the latch-in relay are calculated by the proposed method and the conventional interpolation one. In the conventional method, only a pair of the first and the final mesh is used for the mesh modification without being divided into zones in the range of the movement of the armature.

On the other hand, in the proposed method, we can use the suitable mesh for the calculation due to the division of the zone in the range of the movement of the armature into several sub zones.

\subsection{Analysis Conditions}

The analyzed model for the static torque calculation is the same as the model as shown in Fig. 3. The range of rotation angle of the armature is from $-3.5^{\circ}$ to $3.5^{\circ}$. The exiting current applied to the coil is 304.2 AT. The magnetization of the permanent magnet is $0.86 \mathrm{~T}$. The magnetic nonlinearity of the stator and the armature is taken into account using the B-H curve as shown in Fig. 5 .

\subsection{Results and Discussion}

Fig. 8 shows the static torque characteristics. It is found that the torque calculated by the conventional

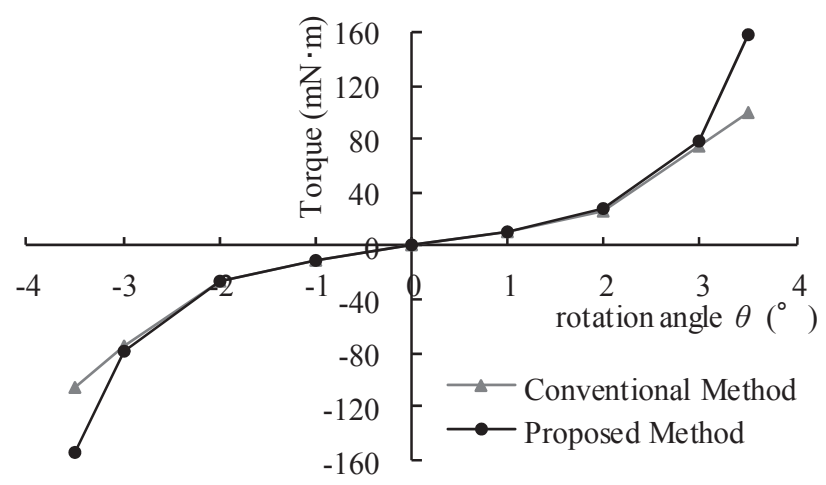

(a) without exiting current

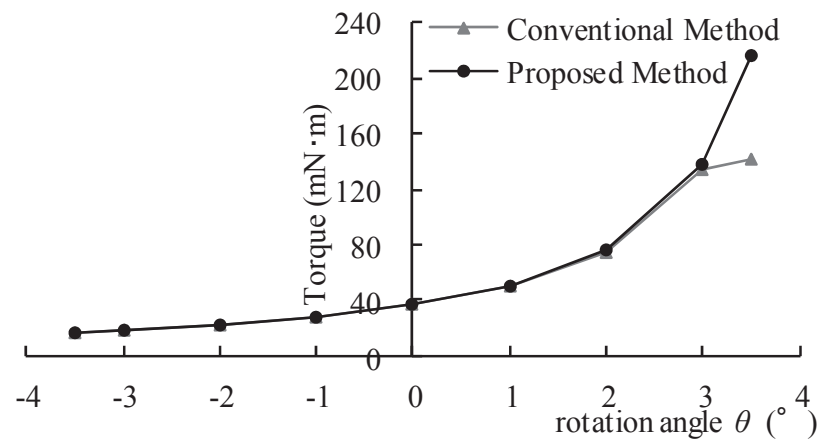

(b) with exiting current

Fig. 8. Static torque characteristics. method agree well with the torque calculated by the proposed method when the rotation angle is from $-2.0^{\circ}$ to $2.0^{\circ}$. However, it is found that the torque calculated by the proposed method differs substantially from the one calculated by the conventional method at the rotation angle $3.5^{\circ}$ in spite of the exiting current. It is confirmed that the torque calculated by the proposed method is good through the comparison of the results calculated using a fine mesh.

Consequently, the proposed method made it possible to calculate the torque accurately when the gap between the stator and the armature is greatly narrow.

\section{Effect of Magnetization of Permanent Magnet on Dynamic Characteristics}

\subsection{Analysis Conditions}

In this section, we discuss the effect of the magnetization of the permanent magnet on dynamic characteristics. The analysis is executed by changing the magnetization of the permanent magnet $M$ to 0.430 , 0.645 and 0.860 . The analysis conditions except the magnetization of the permanent magnet are in Table 2.

\subsection{Results and Discussion}

Fig. 9 shows the time variations of exiting current. From this figure and rotation angle of the armature as shown in Fig. 11, it is found that the time variations of exiting current are affected by the rotation angle of the armature.

Fig. 10 shows the time variations of torque acting on the armature. From this figure, it is found that the torque at the start and the end of operation decreases as $M$ decreases.

Fig. 11 shows the time variations of rotation angle. It is found that the operation time of armature is approximately $10 \mathrm{~ms}$ regardless of $M$.

Fig. 12 shows distributions of flux density vectors at $M=0.86$. From this figure, it is confirmed that the flux density vectors are large near the gap between the stator and the armature.

Table 3 shows the discretization data and CPU time.

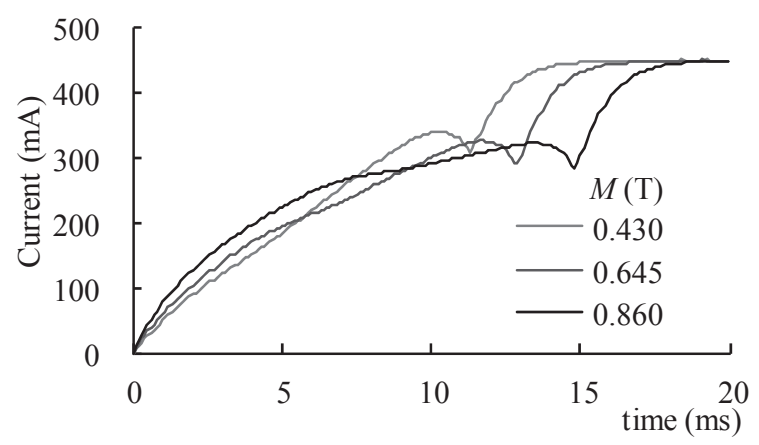

Fig. 9. Time variations of exiting current. 


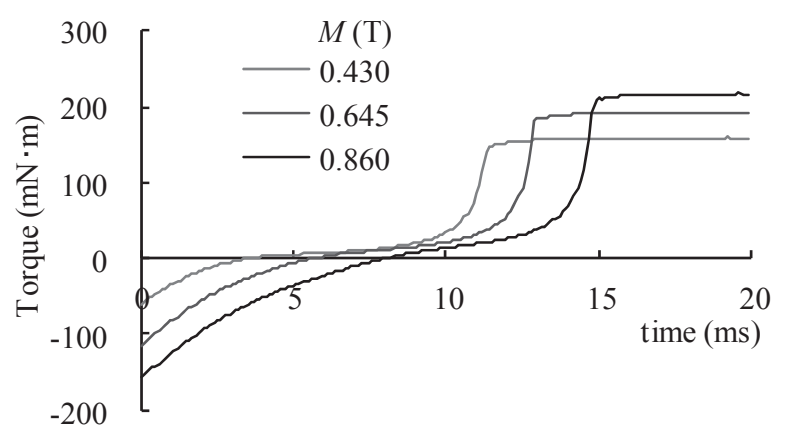

Fig. 10. Time variations of torque.

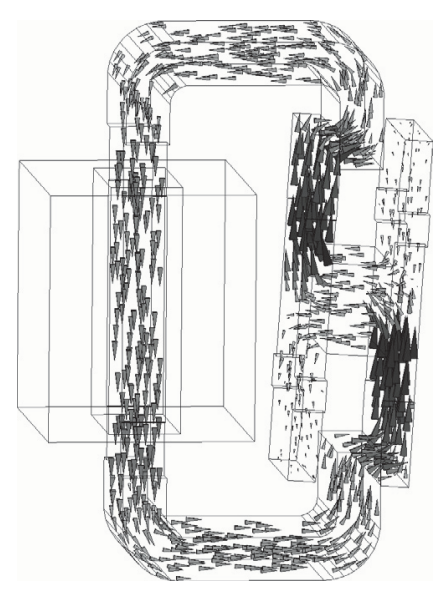

(a) initial state

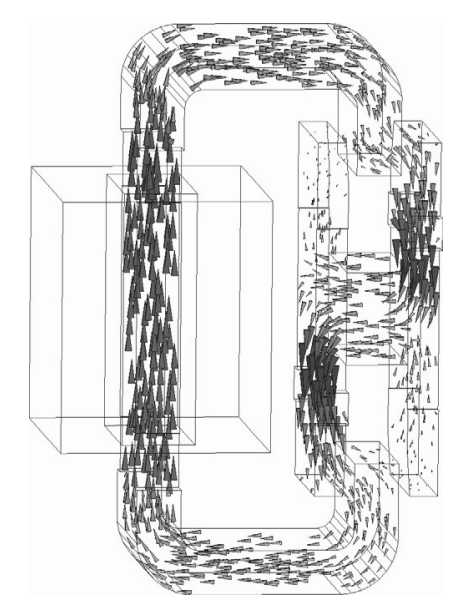

(b) middle state

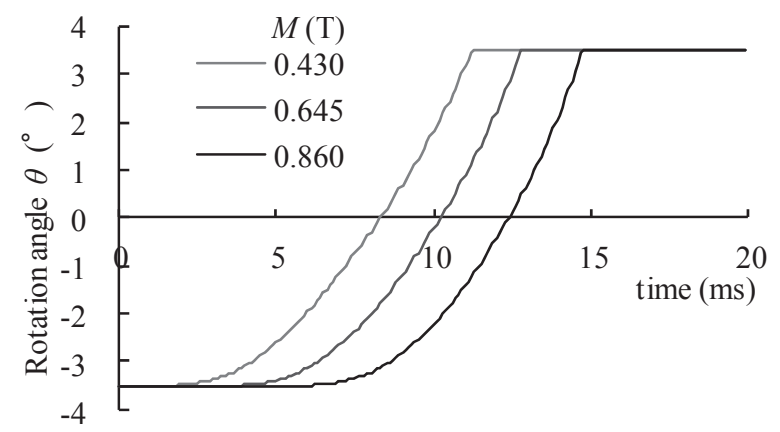

Fig. 11. Time variations of rotation angle.

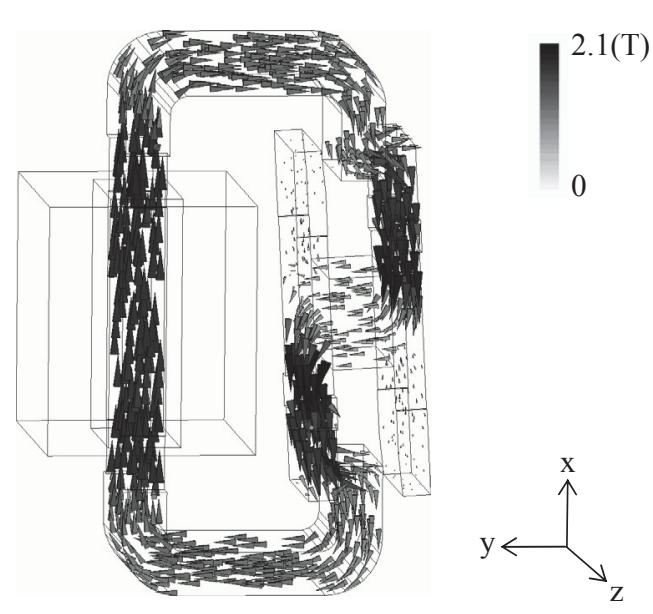

(c) final state

Fig. 12. Distributions of flux density vectors. $(M=0.86)$.

Table 3 Discretization data and CPU time $(M=0.86)$

\begin{tabular}{c|r|r|r}
\hline & \multicolumn{1}{|c|}{ zone I } & \multicolumn{1}{c}{ zone II } & \multicolumn{1}{c}{ zone III } \\
\hline Number of elements & $2,245,800$ & $2,245,344$ & $2,244,432$ \\
\hline Number of nodes & 380,688 & 380,534 & 380,226 \\
\hline Number of edges & $2,639,073$ & $2,638,309$ & $2,636,781$ \\
\hline Number of time steps & \multicolumn{3}{|c}{200} \\
\hline CPU time (hours) & \multicolumn{3}{|c}{48.2} \\
\hline
\end{tabular}

Computer used: Intel Core i7 $(3.40 \mathrm{GHz})$

\section{Conclusion}

In this paper, we proposed a new mesh modification method using the 3-D FEM employing the multi-mesh and the interpolation. The dynamic behavior of a latch-in relay was analyzed using this method, and the effectiveness of this method was clarified.

This method made it possible to modify mesh appropriately according to the rotation angle of the armature and to obtain analysis results accurately.

\section{References}

[1] S. Ito and Y. Kawase, Computer Aided Engineering of Electric and Electronic Apparatus Using Finite Element Method, Morikita Publishing, 2000, (in Japanese).

[2] Y. Kawase, O. Miyatani, T. Yamaguchi and S. Ito, "Numerical Analysis of Dynamic Characteristics of Electromagnets Using 3-D Finite Element Method with Edge Elements", IEEE Trans. Magnetics, Vol. 30, No. 5, pp. 3248-3251, 1994.

[3] S. Suzuki, Y. Kawase, T. Yamaguchi, S. Toyama, K. Hirata and T. Ota, "Dynamic Characteristics Analysis of Circuit Breaker with Oil Dashpot Employing Improved Multi-Mesh Modification Method", The $18^{\text {th }}$ Int. Conf. Computation of Electromag. Fields, PA6.12, 2011.

[4] A. Kameari, "Local force calculation in 3D FEM with edge elements", Int. J. Applied Electromag. in Materials 3, pp. 231-240, 1993. 